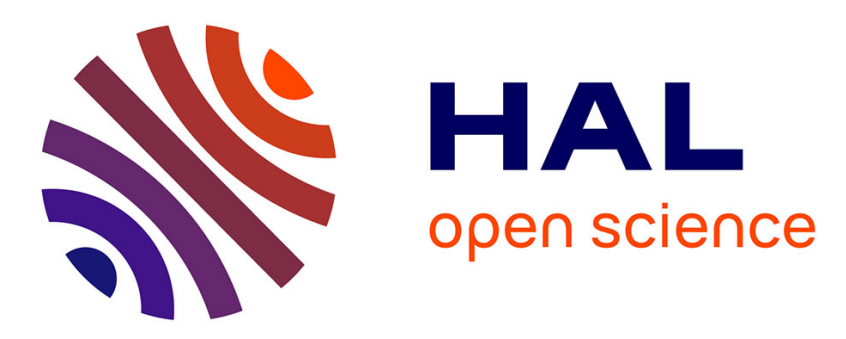

\title{
Plasma inhomogeneities near the electrodes of a capacitively-coupled radio-frequency discharge containing dust particles
}

Hagop Tawidian, Maxime Mikikian, Lénaïc Couëdel, Thomas Lecas

\section{- To cite this version:}

Hagop Tawidian, Maxime Mikikian, Lénaïc Couëdel, Thomas Lecas. Plasma inhomogeneities near the electrodes of a capacitively-coupled radio-frequency discharge containing dust particles. European Physical Journal: Applied Physics, 2011, 56, pp.24018. 10.1051/epjap/2011110163 . hal-00613350

\section{HAL Id: hal-00613350 https://hal.science/hal-00613350}

Submitted on 2 Nov 2011

HAL is a multi-disciplinary open access archive for the deposit and dissemination of scientific research documents, whether they are published or not. The documents may come from teaching and research institutions in France or abroad, or from public or private research centers.
L'archive ouverte pluridisciplinaire HAL, est destinée au dépôt et à la diffusion de documents scientifiques de niveau recherche, publiés ou non, émanant des établissements d'enseignement et de recherche français ou étrangers, des laboratoires publics ou privés. 


\title{
Plasma inhomogeneities near the electrodes of a capacitively- coupled radio-frequency discharge containing dust particles
}

\author{
H. Tawidian ${ }^{\mathrm{a}}$, M. Mikikian ${ }^{\mathrm{b}}$, L. Couëdel ${ }^{\mathrm{c}}$, and T. Lecas \\ GREMI, Groupe de Recherches sur l'Energétique des Milieux Ionisés, UMR 6606, CNRS/Université d'Orléans, \\ 14 rue d'Issoudun, BP6744, 45067 Orléans Cedex 2, France
}

Received: 15 April 2011 / Received in final form: 25 July 2011 / Accepted: 3 August 2011

Published online: 28 October 2011 - (C) EDP Sciences 2011

\begin{abstract}
Small plasma spheroids are evidenced and analyzed in front of the electrodes of a capacitivelycoupled radio-frequency discharge in which dust particles are growing. These regions are characterized by a spherical shape, a slightly enhanced luminosity and are related to instabilities induced by the presence of dust particles. Several types of behaviors are identified and particularly their chaotic appearance or disappearance and their rotational motion along the electrode periphery. Correlations with the unstable behavior of the global plasma glow are performed. These analyses are obtained thanks to high-speed imaging which is the only diagnostics able to evidence these plasma spheroids.
\end{abstract}

\section{Introduction}

Solid particles from a few nanometers to centimeters can be found in laboratories and industrial plasma processes. In the late 1980s, dust particle formation was discovered in industrial reactors $[1,2]$ and became a huge problem, particularly in microelectronics. Indeed, these particles can fall on wafers and affect semiconductor devices performances. Since then, scientists and engineers involved in this field must take into account this potential problem. Lately, with the big achievement concerning electronic devices miniaturization, these dust particles could be used in many industrial applications related to nanotechnology. Dusty plasmas $[3,4]$ can also be found in astrophysical environments [5], like almost everywhere (comet tails, planetary nebulae, atmospheres [6] and rings) or in fusion devices [7] like the future ITER.

In laboratory reactors, there are many methods to create dust particles, mainly using reactive gases [8-14] or material sputtering [15-18]. In the present work, material sputtering is used to grow a high density of sub-micron dust particles. In the plasma, dust particles acquire a negative charge by capturing electrons. This huge loss of free electrons $[19,20]$ can strongly disturb the plasma stability. It can give birth to unstable behaviors [15] like dust particle growth instabilities (DPGI) [21,22].

\footnotetext{
a e-mail: hagop.tawidian@univ-orleans.fr

b e-mail: maxime.mikikian@univ-orleans.fr

c Present address: Laboratoire de Physique des Interactions Ioniques et Moléculaires, UMR 6633 CNRS/Université de Provence, 13397 Marseille, France
}

In this paper, we analyze a new phenomenon occurring during DPGI and consisting of the appearance of small plasma spheroids in the vicinity of discharge electrodes. These regions are characterized by a slightly enhanced luminosity and seem to appear and disappear in a quite chaotic way. In some conditions, they can rotate regularly along the circumference of the electrodes as recently observed [23]. In the following, we focus more precisely on the chaotic phase related to their appearance.

\section{Experimental setup}

The work presented in this paper is performed in the PKE-Nefedov reactor (Plasma Kristall Experiment) that was designed for microgravity experiments [18,24]. This reactor is a capacitively-coupled radio-frequency (rf at 13.56 $\mathrm{MHz}$ in push-pull mode) discharge in argon. The plasma is created between two electrodes (separated by $3 \mathrm{~cm}$ and with a diameter of $4 \mathrm{~cm}$, surrounded by a guard ring) with a rf power around $3 \mathrm{~W}$ and a pressure around 1.6 mbar (a pressure above $1.5 \mathrm{mbar}$ is required to observe the spheroids). Dust particles are grown by sputtering a polymer layer previously deposited on the electrodes [18] and composed of injected micrometer particles (melamine formaldehyde). At the end of each experiment, the grown dust particles settle on the electrodes. This additional material, mainly composed of carbon, is also sputtered and involved in the growth process. After plasma ignition, we can observe many instabilities due to the dust presence $[25,26]$, like DPGI $[21,22]$ or the heartbeat instability $[27,28]$ consisting of successive contractions and expansions of a central dust-free region named 


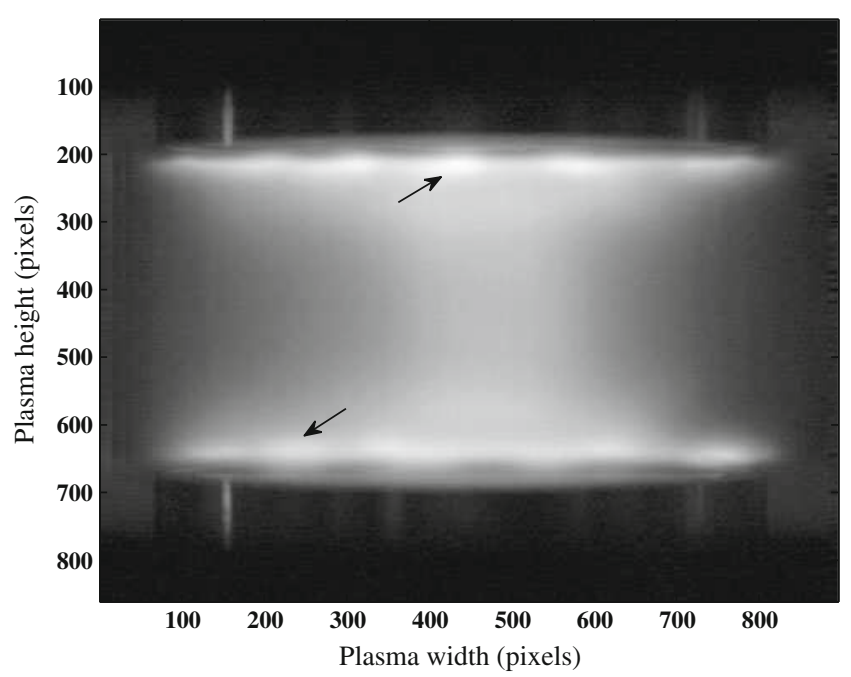

Fig. 1. Plasma glow and detection of spheroids in front of both electrodes. Two plasma spheroids are marked with an arrow.

void. The void region is due to the equilibrium between two major counteracting forces, the electric and ion drag forces [29-31]. All these instabilities are characterized by a strong nonlinear behavior $[28,32]$. To observe the small plasma spheroids appearing during DPGI, we used a highspeed camera "Photron FASTCAM SA5" at 7000 or 10000 frames per second (fps). The camera was positioned directly in front of the reactor at a distance of about $50 \mathrm{~cm}$ to detect and record the plasma glow during the phenomenon. It is noteworthy to mention that, while the other unstable phenomena are quite easily observed, the spheroids discussed in this paper are only visible and detectable thanks to high-speed imaging.

\section{Appearance and behavior of plasma spheroids}

A typical image showing this phenomenon on the plasma glow is presented in Figure 1. This frame is extracted from a movie recorded at 10000 fps during DPGI. Plasma spheroids appear in front of each electrode and are localized on their periphery. They consist of well-defined regions of about a few $\mathrm{mm}$ with a slightly enhanced luminosity. In Figure 1 about six plasma spheroids can be evidenced in front of each electrode. Two of them are marked with an arrow. Due to the angle of view, the top and bottom electrodes appear a little bit tilted. It allows to discriminate plasma spheroids in the foreground from those in the background. In order to reveal more precisely this phenomenon, the regions in the close vicinity of both electrodes $(800 \times 110$ pixels $)$ are extracted from the movie. Plasma luminosity is averaged on the height of each of these regions. Thus, each frame gives two lines (one for each region).

The behavior of the small plasma spheroids in front of both electrodes is analyzed in two different cases. In Figure 2, 1190 frames (170 ms) are processed during a movie recorded at 7000 fps. A spatiotemporal image of
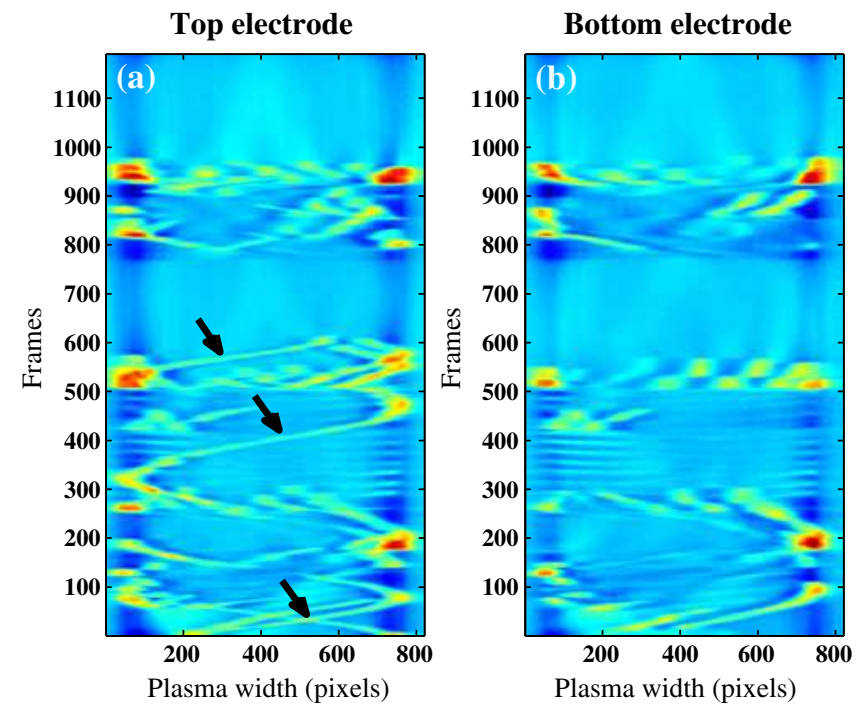

Fig. 2. (Color online) Line profile of plasma glow in front of (a) top and (b) bottom electrodes during 1190 frames. Appearance and disappearance of spheroids during DPGI (brightness variation in false colors from dark blue to red). Some short spheroid paths are marked with arrows.

the plasma spheroid behavior is obtained. Contrast is enhanced by subtracting a temporal average value and by using false colors [27]. From Figure 2, three different regimes related to the plasma spheroids can be evidenced:

- Regime I: No spheroid is observed.

- Regime II: Chaotic appearance and disappearance of spheroids characterized by bright spots.

- Regime III: Persistence of some spheroids that enter in a rotating motion along the circumference of the electrode. This regime corresponds to long spheroids path visible in Figure 2.

The last two regimes are not so strongly separated due to the fact that during the chaotic phase some short spheroids paths exist. These three regimes do not appear always simultaneously in front of both electrodes. From frame 0 to 100 , regime III is observed in front of each electrode but is clearer close to the top electrode (some paths are marked by arrows in Fig. 2a). This regime is followed by regime II until frame 300. Between frames 300 and 420, the regimes in front of each electrode are not similar. Indeed, in Figure 2a we observe regime III (with only one spheroid) while regime $\mathrm{I}$ is evidenced in Figure $2 \mathrm{~b}$. The striations observed in both figures are not related to spheroids and their origin will be explained later in this paper. Two other regimes I are clearly observed simultaneously in both figures between frames 550 and 770, and later after frame 920 . The other parts of Figure 2 are mainly characterized by regime II and very short spheroids paths. In order to correlate the observed regimes with DPGI, the evolution of the central plasma glow is analyzed. It is performed by taking the plasma glow profile along the horizontal axis in between the two electrodes for each frame (Fig. 3). The vertical axis of symmetry of the plasma is 


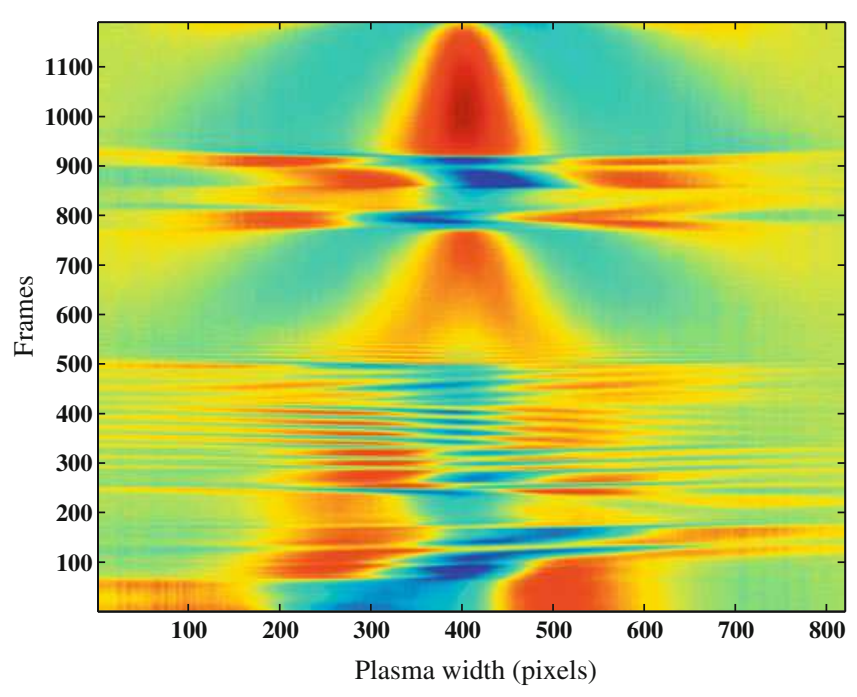

Fig. 3. (Color online) Plasma glow profile along the horizontal axis in between the two electrodes during DPGI (in false colors from dark blue to red).

around 400 on the $x$-axis. It clearly appears that during DPGI the plasma luminosity can strongly vary not only in the center but also toward plasma, and electrode, edges (left and right). Different time scales can also be evidenced with fast and slow changes. By comparing Figures 2 and 3 , several correlations can be made. When the plasma glow changes are mainly concentrated near the discharge center (between frames 550 and 770 or 920 and 1190 in Fig. 3) it corresponds principally to regime I near the electrodes but also to a lesser extent, to some short spheroids paths (Fig. 2). When the plasma behavior in Figure 3 is much more chaotic with off-centered changes (between 0 and 300 or 770 and 920), it corresponds in Figure 2 either to the chaotic appearance of the spheroids (regime II), or to their rotation (regime III). It confirms that regimes II and III can be confused due to the existence of some spheroids paths during the chaotic phase. The phase in between frames 300 and 420 in Figure 3 is characterized by very fast changes in the plasma emission. It explains the striations observed in Figure 2 at the same time.

In the second case we studied, the camera speed was fixed at $10000 \mathrm{fps}$. The spheroids are present all the time close to the top electrode, while many regimes occur near the bottom electrode. These results are shown in Figure 4 obtained using the same procedure as in Figure 2. For the top electrode the spheroids paths clearly appear (Fig. 4a). In the beginning three rotating spheroids (regime III) are detected and keep turning for several rounds at a speed of about $4 \mathrm{~m} \mathrm{~s}^{-1}$. At frame 720 this regime is interrupted by a chaotic phase with many spheroids appearing and disappearing quickly (regime II). This regime stops at frame 1000 where a three spheroid system is back again until the second chaotic phase at frame 1400. Once again regime III is back but the number of spheroids is reduced to one, turning around the top electrode. A second spheroid joins it at frame 1900 till the end of the video
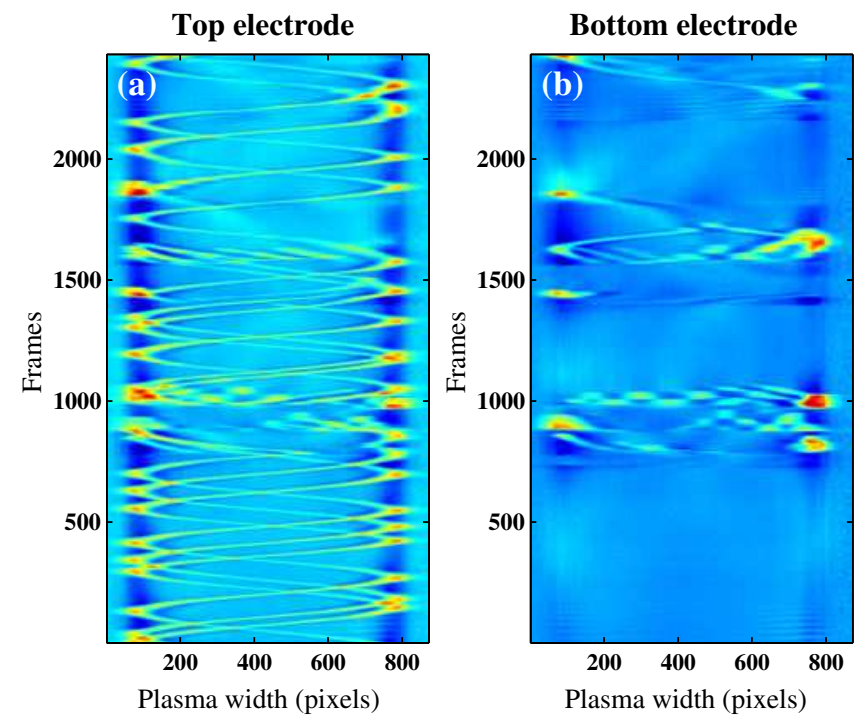

Fig. 4. (Color online) Line profile of plasma glow in front of (a) top and (b) bottom electrodes during 2431 frames. Appearance and disappearance of spheroids during DPGI (the brightness variation in false colors from dark blue to red).

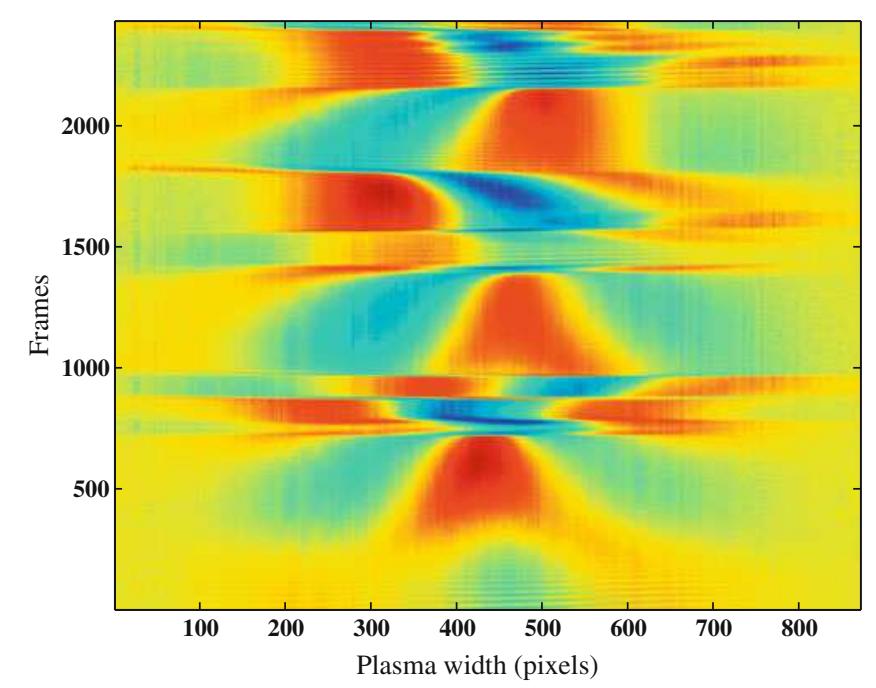

Fig. 5. (Color online) Plasma glow profile along the horizontal axis in between the two electrodes during DPGI (in false colors from dark blue to red).

recording. Concerning the bottom electrode (Fig. 4b), the spheroids just appear during the chaotic phase. When a nice rotation regime is observed near the top electrode, nothing is observed close to the bottom one except at the end (around frame 2200). By comparing the phenomena happening close to both electrodes with the luminosity of the middle region of the plasma (Fig. 5), same behaviors as in Figures 2 and 3 are observed. Slow and central changes in the plasma luminosity (for example, between frames 1000 and 1400) correspond to either no spheroid (Fig. 4b) or rotating spheroids (Fig. 4a). Fast and off-centered plasma changes (for example, between frames 720 and 1000) are related to chaotic regimes for the spheroids. 


\section{Discussion and conclusion}

In this paper, we have characterized and analyzed a new phenomenon occurring during DPGI and consisting of the appearance of small plasma spheroids in front of the discharge electrodes. In the studied cases, different regimes have been clearly identified and correlated with the variation of the plasma luminosity in the discharge central part. We found that when the plasma changes are mainly located in the close discharge center, regime I (no spheroid) or III (rotating spheroids) is observed in front of the electrodes. Regime III (chaotic appearance and disappearance of the spheroids) appears when plasma changes in between the electrodes are fast and more off-centered.

The origin of these plasma spheroids is not well elucidated. Their appearance in front of the electrodes seems to be correlated with DPGI as they have been observed only during or after DPGI. In this paper we focused on the chaotic appearance of the spheroids which occurs only during DPGI. When plasma spheroids are observed after DPGI, only regime III, with spheroids rotating regularly at a few $\mathrm{m} \mathrm{s}^{-1}$, has been observed until now (Carousel instability [23]). In this last case, DPGI seem to be finished or are no more detectable.

These spheroids are observed along the periphery of the electrodes indicating that the guard ring surrounding the electrode can play a role in this phenomenon. However, these spheroids have always been observed in dusty plasma conditions with a high density of sub-micron dust particles and a quite high pressure ( $>1.5$ mbar). We can assume that the strong disturbance induced by the dust particle presence can trigger the spheroid appearance or rotation. Thus, this behavior occurs preferentially in regions where electrical inhomogeneities exist as close to the guard rings. A similar phenomenon has been described in [33]. In this last case, the disturbance of the plasma equilibrium is not induced by dust particles but by the introduction of a glass tube into the discharge.

The authors would like to thank L. Boufendi for supporting this project. B. Dumax is acknowledged for electronic support and J.-M. Bauchire and H. Rabat for providing the high-speed camera. The PKE-Nefedov chamber has been made available by the Max-Planck-Institute for Extraterrestrial Physics, Germany, under the funding of DLR/BMBF under Grant No. 50WM9852.

\section{References}

1. R.M. Roth, K.G. Spears, G.D. Stein, G. Wong, Appl. Phys. Lett. 46, 253 (1985)

2. G.S. Selwyn, J. Singh, R.S. Bennett, J. Vac. Sci. Technol. A 7, 2758 (1989)

3. P.K. Shukla, B. Eliasson, Rev. Mod. Phys. 81, 25 (2009)

4. M. Mikikian, L. Couëdel, M. Cavarroc, Y. Tessier, L. Boufendi, Eur. Phys. J. Appl. Phys. 49, 13106 (2010)
5. C.K. Goertz, Rev. Geophys. 27, 271 (1989)

6. E. Sciamma-O'Brien, N. Carrasco, C. Szopa, A. Buch, G. Cernogora, Icarus 209, 704 (2010)

7. S.I. Krasheninnikov et al., Plasma Phys. Control. Fusion 50, 124054 (2008)

8. J. Berndt, E. Kovačević, I. Stefanović, O. Stepanovic, S.H. Hong, L. Boufendi, J. Winter, Contrib. Plasma Phys. 49, 107 (2009)

9. M. Mikikian, M. Cavarroc, L. Couëdel, Y. Tessier, L. Boufendi, Pure Appl. Chem. 82, 1273 (2010)

10. J. Benedikt, J. Phys. D: Appl. Phys. 43, 043001 (2010)

11. J. Pereira, V. Massereau-Guilbaud, I. Géraud-Grenier, A. Plain, J. Appl. Phys. 103, 033301 (2008)

12. M. Cavarroc, M. Mikikian, Y. Tessier, L. Boufendi, Phys. Rev. Lett. 100, 045001 (2008)

13. J. Berndt, E. Kovačević, I. Stefanović, L. Boufendi, J. Appl. Phys. 106, 063309 (2009)

14. S. Dap, D. Lacroix, F. Patisson, R. Hugon, L. de Poucques, J. Bougdira, New J. Phys. 12, 093014 (2010)

15. D. Samsonov, J. Goree, Phys. Rev. E 59, 1047 (1999)

16. C. Dominique, C. Arnas, J. Appl. Phys. 101, 123304 (2007)

17. A. Michau, G. Lombardi, L.C. Delacqua, M. Redolfi, C. Arnas, X. Bonnin, K. Hassouni, Plasma Phys. Control. Fusion 52, 124014 (2010)

18. M. Mikikian, L. Boufendi, A. Bouchoule, H.M. Thomas, G.E. Morfill, A.P. Nefedov, V.E. Fortov, the PKE-Nefedov Team, New J. Phys. 5, 19 (2003)

19. W.W. Stoffels, E. Stoffels, G.M.W. Kroesen, F.J. de Hoog, J. Appl. Phys. 78, 4867 (1995)

20. H. Kersten, H. Deutsch, M. Otte, G.H.P.M. Swinkels, G.M.W. Kroesen, Thin Solid Films 377-378, 530 (2000)

21. M. Mikikian, M. Cavarroc, L. Couëdel, L. Boufendi, Phys. Plasmas 13, 092103 (2006)

22. M. Mikikian, L. Couëdel, M. Cavarroc, Y. Tessier, L. Boufendi, IEEE Trans. Plasma Sci. 36, 1012 (2008)

23. M. Mikikian, L. Couëdel, Y. Tessier, L. Boufendi, IEEE Trans. Plasma Sci. (in press), DOI: http://dx.doi.org/ 10.1109/TPS.2011.2155675

24. A.P. Nefedov et al., New J. Phys. 5, 33 (2003)

25. S.K. Zhdanov et al., New J. Phys. 12, 043006 (2010)

26. L. Couëdel, M. Mikikian, A.A. Samarian, L. Boufendi, Phys. Plasmas 17, 083705 (2010)

27. M. Mikikian, L. Couëdel, M. Cavarroc, Y. Tessier, L. Boufendi, New J. Phys. 9, 268 (2007)

28. M. Mikikian, L. Couëdel, M. Cavarroc, Y. Tessier, L. Boufendi, Phys. Rev. Lett. 105, 075002 (2010)

29. J. Goree, G.E. Morfill, V.N. Tsytovich, S.V. Vladimirov, Phys. Rev. E 59, 7055 (1999)

30. Z. Hu, Y. Chen, X. Zheng, F. Huang, G.-F. Shi, M.Y. Yu, Phys. Plasmas 16, 063707 (2009)

31. C. Schmidt, O. Arp, A. Piel, Phys. Plasmas 18, 013704 (2011)

32. M. Mikikian, M. Cavarroc, L. Couëdel, Y. Tessier, L. Boufendi, Phys. Rev. Lett. 100, 225005 (2008)

33. J. Schulze, D. Luggenhölscher, U. Czarnetzki, IEEE Trans. Plasma Sci. 36, 1402 (2008) 